\title{
Successful fibrinolytic and therapeutic hypothermic management of cardiac arrest following massive pulmonary embolism
}

\author{
Eunsil Ko, Jeong Hoon Lee, Minjung Kathy Chae, Tae Rim Lee, \\ Min Seob Sim, Tae Gun Shin, Won Chul Cha, Ik Joon Jo, \\ Keun Jeong Song, Joong Eui Rhee, Yeon Kwon Jeong
}

Department of Emergency Medicine, Samsung Medical Center, Sungkyunkwan University School of Medicine, Seoul, Korea

Massive pulmonary embolism (MPE) with hemodynamic instability is a clinical condition with a poor prognosis and high mortality rates. There are no definitive treatment options for cardiac arrest due to MPE. A 52-year-old female presented at our emergency department with cardiac arrest, and a 62-year-old female presented after achieving return of spontaneous circulation of cardiac arrest from a local hospital, respectively. In each case, computed tomographic pulmonary angiography after return of spontaneous circulation demonstrated heavy burdens of pulmonary embolism in the pulmonary arteries. We immediately started therapeutic hypothermia and fibrinolytic therapy. They were transferred to the thoracic surgery and cardiology departments respectively, and then discharged with a cerebral performance categories scale score of 1. In summary, we report two cases of out-of-hospital cardiac arrest due to MPE in which fibrinolytic therapy was successfully combined with therapeutic hypothermia.

Keywords Heart arrest; Fibrinolysis; Pulmonary embolism; Hypothermia, induced

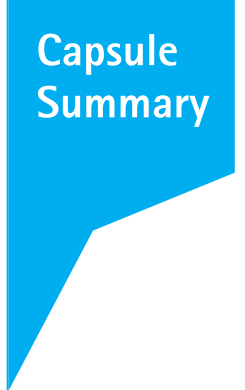

elSSN: 2383-4625

Received: 12 March 2015

Revised: 11 April 2015

Accepted: 14 April 2015

Correspondence to: Tae Rim Lee Department of Emergency Medicine, Samsung Medical Center,

Sungkyunkwan University School of Medicine, 81 Irwon-ro, Gangnam-gu, Seoul 135-710, Korea

E-mail:kimmy0813@hanmail.net

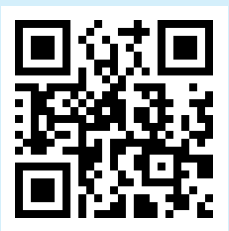

How to cite this article:

Ko E, Lee JH, Chae MK, Lee TR, Sim MS, Shin TG, Cha WC, Jo IJ, Song KJ, Rhee JE, Jeong YK. Successful fibrinolytic and therapeutic hypothermic management of cardiac arrest following massive pulmonary embolism. Clin Exp Emerg Med 2015;2(3): 193-196.

This is an Open Access article distributed under the terms of the Creative Commons Attribution Non-Commercial License (http:// creativecommons.org/licenses/by-nc/3.0/). 


\section{INTRODUCTION}

Pulmonary thromboembolism (PTE) is a relatively rare cause of cardiac arrest. ${ }^{1}$ Even when return of spontaneous circulation (ROSC) is achieved, mortality related to cardiac arrest due to PTE is high. ${ }^{2}$ In the 2010 American Heart Association and the European Resuscitation Council guidelines, there are no definitive treatment options for cardiac arrest due to PTE. ${ }^{3,4}$ Although surgical embolectomy is considered the most effective method to treat PTE, emergency surgery after PTE-related cardiac arrest is associated with high mortality. ${ }^{5}$ We report two successfully resuscitated cases of cardiac arrest due to massive PTE by using recombinant tissuetype plasminogen activator (rTPA) and therapeutic hypothermia. We present these cases with a literature review.

\section{CASE REPORTS}

\section{Case 1}

A 52-year-old female was transferred to our emergency department (ED) from a local orthopedic clinic, presenting with cardiac arrest. She previously had a right ankle fracture and lumbar vertebral compression fracture due to a traffic accident seven days prior to cardiac arrest. She was found unconscious with a pulse in the bathroom of a local clinic after complaining of chest discomfort and dyspnea. On arrival in the ED, her initial rhythm was pulseless electrical activity. The estimated no-flow time and lowflow time were unknown and three minutes, respectively. After achieving ROSC, blood pressure (BP) was $49 / 35 \mathrm{mmHg}$ and heart rate was $135 \mathrm{bpm}$. Her mental status was comatose with fully dilated pupils. However, there were sluggish light reflexes, corneal reflexes and spontaneous respirations. After using norepinephrine $(120 \mu \mathrm{g} / \mathrm{min})$ and dopamine $(30 \mu \mathrm{g} / \mathrm{kg} / \mathrm{min})$, BP was stabilized at $129 / 93 \mathrm{mmHg}$. Oxygen saturation was $100 \%$ with ventilation with $60 \% \mathrm{FiO}_{2}$. Computed tomographic pulmonary angiography showed heavy burden of pulmonary embolism in the bilateral pulmonary arteries and branches (Fig. 1A, B). On echocardiography, a D-shaped left ventricle was observed during systole and diastole. Within two hours of arrival at the ED, she was admitted to the ED intensive care unit, and we started therapeutic hypothermia using a commercial temperature regulation system (Arctic Sun Temperature Management System; Medivance, Louisville, CO, USA) with our hospital protocol (rapid cooling to $33^{\circ} \mathrm{C}$, 24hour maintenance, and $0.15^{\circ} \mathrm{C} / \mathrm{hr}$ rewarming to $36.5^{\circ} \mathrm{C}$ ). Midazolam and fentanyl were used for sedation during therapeutic hypothermia. We also administrated $100 \mathrm{mg}$ of rTPA over two hours, with a loading dose of 60 unit $/ \mathrm{kg}$ followed by a continuous intravenous infusion of unfractionated heparin (12 unit $/ \mathrm{kg} / \mathrm{hr}$ ). While
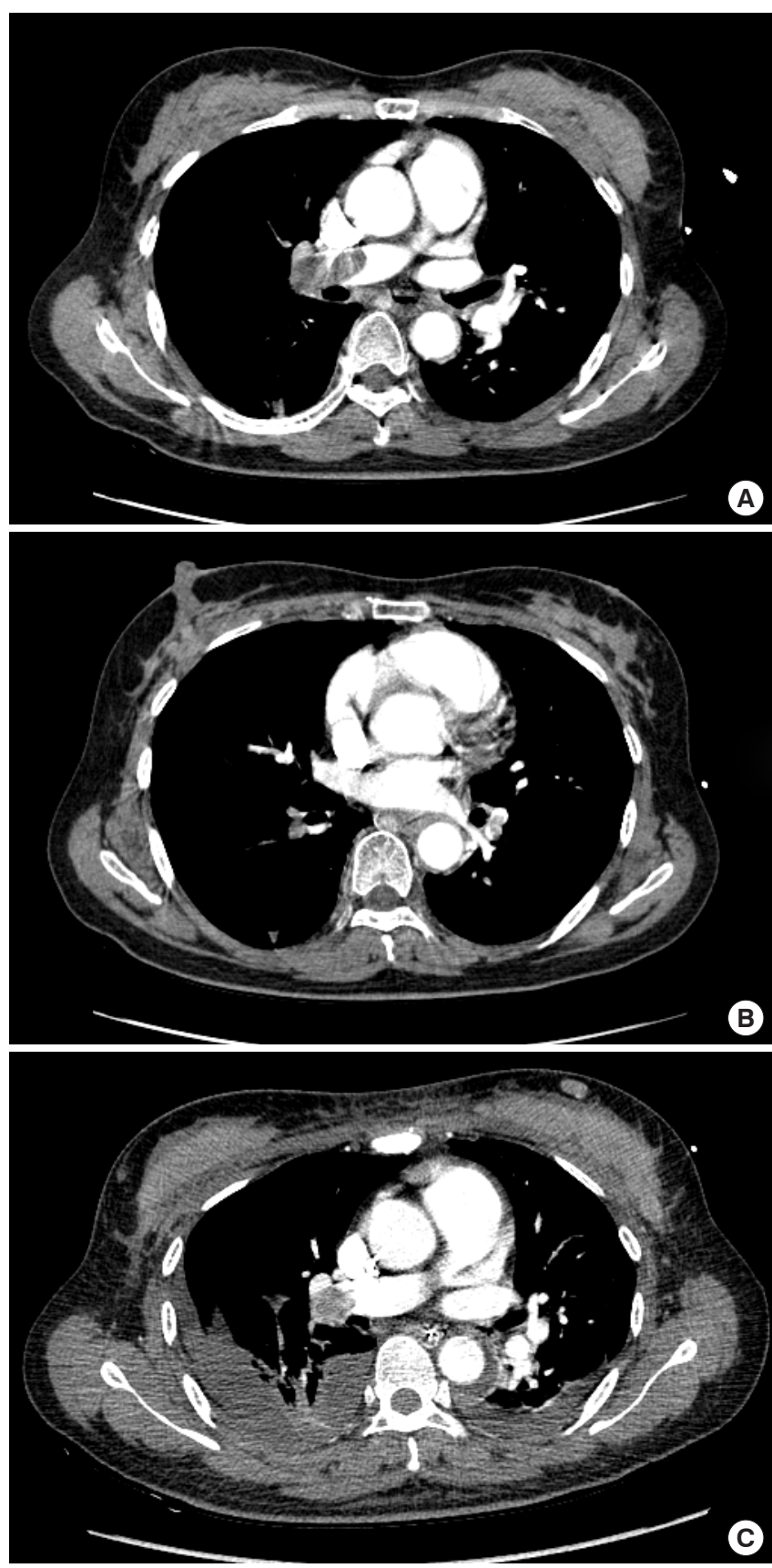

Fig. 1. Computed tomographic pulmonary angiographic images of case 1. The initial angiography showed thrombi in the right main distal pulmonary artery (A) and the left pulmonary arteries (B). (C) Follow-up angiography showed regression of thrombi in the right main distal pulmonary artery.

conducting therapeutic hypothermia, no bleeding complications were observed. On her third hospitalized day (HD3), she was able to follow simple commands and vital signs were stable without inotropes. The follow-up computed tomographic angiography at HD3 showed that the burden of pulmonary embolism remained in the right main pulmonary artery (Fig. 1C). Right ventricular 
systolic pressure, estimated by the maximum velocity of tricuspid regurgitation and right atrium pressure, was normalized (from $>58$ to $24.1 \mathrm{mmHg}$ ) on echocardiography. She was transferred to the thoracic surgery department to undergo a pulmonary artery embolectomy. At HD28, she was discharged with a cerebral performance categories scale score of 1 , without neurological deficits.

\section{Case 2}

A 62-year-old female was transferred to our ED from a local tertiary hospital after achieving ROSC after cardiac arrest. She had had a radical hysterectomy and bilateral salpingo-oophorectomy due to cervical cancer 20 days prior to the cardiac arrest. Her collapse was witnessed, and her initial heart rhythm was ventricular fibrillation. She achieved ROSC after 50 minutes of advanced life support. When she arrived in our ED four hours after cardiac arrest, her BP and heart rate were 129/66 mmHg and 120 bpm with norepinephrine $(20 \mu \mathrm{g} / \mathrm{min})$, but she was comatose. Her light reflex, corneal reflex, and spontaneous respirations were intact. Computed tomographic pulmonary angiography showed bilateral pulmonary artery thromboembolism (Fig. 2A), with a D-shaped left ventricle during systole and diastole visible on echocardiography. After admission to the ED intensive care unit within two hours of arrival, we started therapeutic hypothermia using a conventional method (a commercial cold blanket, as well as $4^{\circ} \mathrm{C}$ cold saline bladder irrigation or traditional ice packs placed on the patient's groin, armpits, and neck and head) as per our hospital protocol. Midazolam and fentanyl were used for sedation during therapeutic hypothermia. We administered $100 \mathrm{mg}$ of rTPA over two hours, with a loading dose of $60 \mathrm{unit} / \mathrm{kg}$ followed by a continuous intravenous infusion of unfractionated heparin (12 unit/kg/hr). At two hours after administration of rTPA, massive bleeding was observed via Nasogastric (NG)-tube. We started transfusion and consulted for endoscopy. The endoscopy showed a gastric ulcer with bleeding at the stomach fundus. The bleeding was stabilized after one day with conservative treatment. At HD5, her mental status was recovered to nearly alert. Follow-up computed tomographic angiography showed minimal residual pulmonary emboli (Fig. 2B, C). Right ventricular systolic pressure was normalized (from 41.72 to $29 \mathrm{mmHg}$ ) on echocardiography. At HD7, she was extubated and transferred to the cardiology department for an inferior vena cava filter insertion. At HD27, she was discharged with a cerebral performance categories scale score of 1 .

\section{DISCUSSION}

Massive pulmonary embolism (MPE) with hemodynamic instability is a clinical condition with a poor prognosis and a mortality
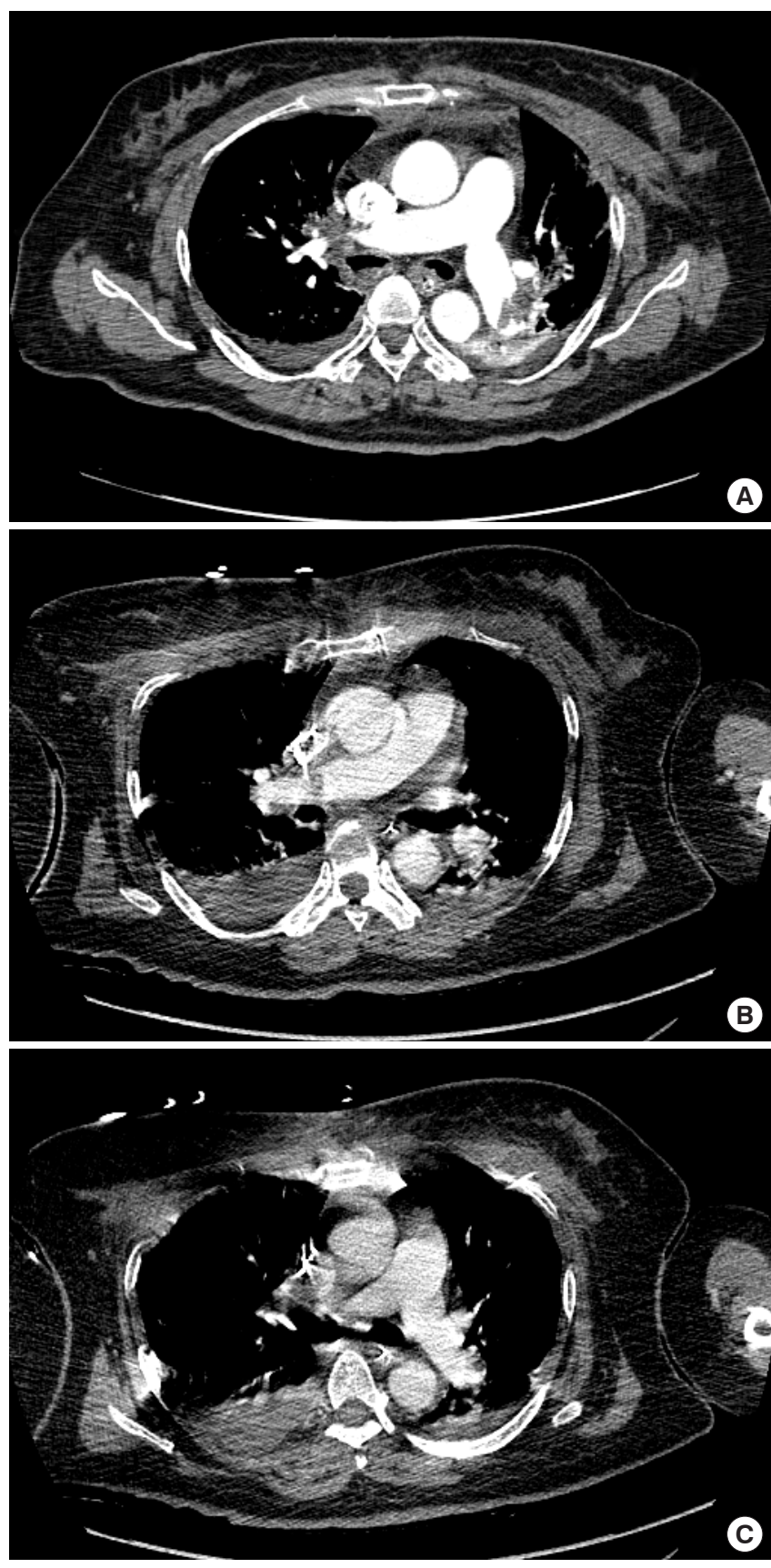

Fig. 2. Computed tomographic pulmonary angiographic images of case 2. (A) The initial angiography showed thrombi in the pulmonary arteries. $(B, C)$ Follow-up angiography showed minimal residual thrombi in the pulmonary arteries.

rate greater than $50 \% .{ }^{6}$ Current guidelines recommend aggressive treatment with systemic fibrinolytic therapy, catheter embolectomy, or surgical embolectomy, depending on the local situation. ${ }^{3,4}$ Fibrinolytic therapy remains the most commonly used treatment in hypotensive patients presenting with a MPE in the ED. Catheter embolectomy or surgical embolectomy can be used as an alternative MPE treatment method when fibrinolytic treatment 
is contraindicated or fails. ${ }^{2,3}$

When cardiac arrest due to acute MPE occurs, the risk of death increases up to $70 \% .{ }^{2}$ In spite of achieving ROSC, cardiac arrest due to MPE is often fatal because it results from mechanical obstruction of the pulmonary arterial tree by thrombi. Although fibrinolytic therapy is not recommended for undifferentiated cardiac arrest, it should be considered for cardiac arrest patients diagnosed with MPE. ${ }^{3}$ rTPA has been preferred as a fibrinolytic agent. According to one small case series reported in 2014, surgical embolectomy with percutaneous cardiopulmonary support is considered the preferred method for MPE-induced cardiac arrest. However, overall mortality remained high in patients who underwent cardiopulmonary resuscitation (CPR) for more than 15 minutes. ${ }^{7}$

Therapeutic hypothermia is recommended as a neuro-protective strategy after cardiac arrest. ${ }^{8}$ There are no randomized trials guiding the use of fibrinolysis with hypothermia in cardiac arrest survivors following MPE. It has been reported that a hypothermia-induced coagulation disorder could increase the risk of serious adverse events, ${ }^{4}$ but it is unclear if bleeding complications occur more often in cardiac arrest patients treated with therapeutic hypothermia. In a recent systematic review and meta-analysis, the risk of bleeding was not significantly higher for patients treated with therapeutic hypothermia. ${ }^{9}$ Relative contraindications to fibrinolytic therapy include prolonged CPR (> 10 minutes) and major surgery within three weeks prior to cardiac arrest. ${ }^{3}$ However, current guidelines recommend that physicians weigh the relative benefits of fibrinolysis on a case-by-case basis. ${ }^{3}$ In our cases, the cardiac arrest survivor with prolonged CPR was diagnosed with MPE and then had fibrinolytic therapy using rTPA with therapeutic hypothermia. Although gastric ulcer bleeding was observed for the first few hours, it is unclear if this bleeding was made worse by fibrinolytic therapy or therapeutic hypothermia.

In conclusion, we describe two cases of cardiac arrest due to MPE in which fibrinolytic therapy was successfully combined with therapeutic hypothermia. This report suggests that fibrinolysis with hypothermia are feasible in the treatment of MPE with cardiac arrest. However, larger scale and randomized trials are necessary to establish standard therapy for MPE with cardiac arrest.

\section{CONFLICT OF INTEREST}

No potential conflict of interest relevant to this article was reported.

\section{REFERENCES}

1. Kurkciyan I, Meron G, Sterz F, et al. Pulmonary embolism as a cause of cardiac arrest: presentation and outcome. Arch Intern Med 2000;160:1529-35.

2. Kearon C, Akl EA, Comerota A, et al. Antithrombotic therapy for VTE disease: antithrombotic therapy and prevention of thrombosis, 9th ed: american college of chest physicians evidence-based clinical practice guidelines. Chest 2012;141:e419S94S.

3. Jaff MR, McMurtry MS, Archer SL, et al. Management of massive and submassive pulmonary embolism, iliofemoral deep vein thrombosis, and chronic thromboembolic pulmonary hypertension: a scientific statement from the American Heart Association. Circulation 2011;123:1788-830.

4. Deakin CD, Nolan JP, Soar J, et al. European Resuscitation Council Guidelines for Resuscitation 2010 section 4: adult advanced life support. Resuscitation 2010;81:1305-52.

5. Dauphine C, Omari B. Pulmonary embolectomy for acute massive pulmonary embolism. Ann Thorac Surg 2005;79:1240-4.

6. Konstantinides SV. Massive pulmonary embolism: what level of aggression? Semin Respir Crit Care Med 2008;29:47-55.

7. Cho YH, Kim WS, Sung K, et al. Management of cardiac arrest caused by acute massive pulmonary thromboembolism: importance of percutaneous cardiopulmonary support. ASAIO J 2014;60:280-3.

8. Peberdy MA, Callaway CW, Neumar RW, et al. Part 9. Postcardiac arrest care: 2010 American Heart Association Guidelines for Cardiopulmonary Resuscitation and Emergency Cardiovascular Care. Circulation 2010;122:S768-86.

9. Williams TA, McKenzie N, Inoue M. Does therapeutic temperature management after cardiac arrest increase the risk of bleeding? Aust Crit Care 2015 Feb 7. DOI: http://dx.doi.org/10. 1016/j.aucc.2015.01.001 